Rev.MVZ Córdoba 19(2):4045-4046, 2014. ISSN: 0122-0268

\title{
EDITORIAL
}

\section{Virus Chikungunya in Colombia, a simple matter of time?}

\section{¿Virus Chikungunya en Colombia, simple cuestión de tiempo?}

Chikungunya virus (CHIKV) is an RNA alphavirus of the family Togaviridae. The alphaviruses consist of 29 species, including eastern, western, and Venezuelan equine encephalitis viruses among others. CHIKV is transmitted by vector mosquitoes, Aedes aegypti and Aedes albipictus, which are abundant in the South American tropics.

CHIKV was first isolated in 1953 in the serum of a patient in Tanzania during an epidemic of dengue. In a recent dendrogram, this isolate appeared with the name of Ross low psg $(1,2)$. The first clinical report of chikungunya was in Thailand between 1962 and 1964 (3). Between 1960 and 2003 CHIKV reemerged and spread in Southeast Asian countries such as India, Malaysia, Indonesia, Vietnam, and Thailand, among others. A Central African genotype has occurred since 2000 and spread to Europe, Asia, and Australia (4). From the clinical perspective, CHIKV may produce acute, sub-acute, or chronic illness. The acute phase is characterized by an abrupt onset with fever surpassing $39^{\circ} \mathrm{C}$ and severe joint pain. Polyarthralgia, headache, myalgia, back pain, nausea, vomiting, rash, and conjunctivitis are common; the duration of the acute phase is 3 to 10 days (5). Symptoms of CHIKV infection are similar to dengue.

The inevitable arrival of CHIKV in America occurred on 5 December 2013, when the first indigenous case appeared on the Caribbean island Saint Martin in the French West Indies. On 20 December, 50 cases were confirmed on the island of Martinique; in January 2014 CHIKV rapidly disseminated to the islands of Guadeloupe, Saint Barthelemy, Dominica and the British Virgin Islands. (1). In Rio de Janeiro, Brazil, an imported case was reported in a patient who had visited Sumatra, indicating the high potential for spread of the virus by imported patients bitten by local mosquitoes (6).

The vector of CHIKV was initially believed to be only Aedes aegypti; however, the virus was also adapted to Aedes albipictus, Ae. Vittatus, and Anopheles stephensi. The change of vector
El virus Chikungunya (CHIKV) es un ARN alfavirus de la familia Togaviridae. Los alfavirus tienen 29 especies, e incluye los virus de la encefalitis equina oriental, occidental y venezolana entre otros. CHIKV es transmitido por los mosquitos vectores Aedes aegypti y Aedes albopictus los cuales son abundantes en el trópico suramericano.

CHIKV fue aislado por primera vez en 1953 en el suero de un paciente de Tanzania durante una epidemia de dengue. En un dendrograma reciente apareció este aislamiento con el nombre de Ross low psg $(1,2)$. El primer reporte clínico del virus fue en Tailandia entre 1962 y 1964 (3). Entre 1960 y 2003 el virus reemergió y se diseminó en el sudeste asiático en países como India, Malasia, Indonesia, Vietnam y Tailandia entre otros. El genotipo africano centro-oriental ha predominado desde el 2000 con una diseminación hacia Europa, Asia y Australia (4). Desde el punto de vista clínico, CHIKV puede producir una enfermedad aguda, subaguda o crónica. La fase aguda se caracteriza por un comienzo abrupto con fiebre alta superior a los $39^{\circ} \mathrm{C}$ y dolor articular severo. Son comunes la poliartralgia, cefalea, mialgias, dolor de espalda, náuseas, vómitos, rash y conjuntivitis, la duración de la fase aguda puede durar entre 3 y 10 días (5). Los síntomas por infección de CHIKV son similares a los del dengue.

La inevitable llegada a América del CHIKV, sucedió el 5 de diciembre de 2013, cuando apareció el primer caso autóctono en la isla del Caribe San Martin en el archipiélago de las islas francesas del Oeste. El 20 de diciembre 50 casos fueron confirmados en la isla de Martinica y en enero de 2014 se extendió a las islas de Guadalupe, San Bartolomé, Dominicanas e Islas Vírgenes Británicas (1). En Rio de Janeiro Brasil se informó un caso importado de un paciente que había visitado Sumatra, lo que indica el alto potencial de propagación del virus por pacientes importados cuando estos son picados por mosquitos locales (6).

Inicialmente se creyó que el vector de CHIKV era sólo el Aedes aegypti; sin embargo, el virus también se adaptó a Aedes albipictus, Ae. Vittatus 
represents a surprising evolutionary adaptation that involved a mutation in the viral envelope that facilitates replication. The genus Aedes has a wide rural and urban distribution in Colombia and specifically in the Caribbean area, which could facilitate the introduction and subsequent endemicity of CHIKV simply and naturally. It follows that Colombian health authorities should identify aircraft and boats from Venezuela and the Caribbean and fumigate holds before removing cargo and baggage. We do not know the ultimate outcome of competition of CHIKV and Dengue virus in a single vector. Nor do we know whether CHIKV will have an enzootic cycle as in Africa and Asia, or a sylvatic cycle in primates and other vertebrates of the Caribbean region by which it could enter Colombia, as West Nile virus did a few years ago.

The introduction of a new pathogen in Colombia poses more challenges than dengue. The impact of CHIKV in other countries has been worse than dengue, causing lengthy absenteeism from work and disabling symptoms (arthritis, arthralgia) that can last for weeks or months. We do not know if this visiting virus has virulence factors that could transform it into a hemorrhagic virus; we only know that CHIKV is very close to the Asian strains isolated in China in 2012, and the Philippines in 2013.

As seen of other emerging and re-emerging viruses, the arrival of the CHIKV in Colombia is simply a matter of time (currently in Venezuela). We must prepare ourselves for a new differential diagnosis and assume from now on the clinical and ecoepidemiological surveillance. y a Anopheles stephensi. El cambio del vector representa una sorprendente adaptación evolutiva que implicó una mutación en la envoltura del virus lo que facilitó su replicación. El género Aedes sp tiene una amplia distribución rural y urbana en Colombia y específicamente en el área del Caribe, lo que podría facilitar la introducción del virus y la subsecuente endemia de una forma natural y sencilla. En este punto las autoridades sanitarias colombianas deberían prestar atención a los aviones y barcos provenientes de Venezuela y del Caribe, con el fin de fumigar las bodegas antes de sacar la carga y equipajes. No sabemos cómo sería la competencia en un mismo vector de dos virus importantes como el dengue y CHIKV. Tampoco sabemos si el CHIKV tiene un ciclo enzoótico como en África y Asia y si tendrá un ciclo selvático en primates u otros vertebrados de la región Caribe por donde se cree ingresará como lo hizo el virus del Nilo a Colombia hace unos años.

La introducción de un nuevo patógeno en Colombia plantea más retos que el dengue, el impacto de CHIKV en otros países ha sido peor que el dengue, por el largo ausentismo laboral y síntomas incapacitantes (artritis, artralgias) que pueden durar semanas o meses. No conocemos si este virus visitante tendrá factores de virulencia que lo transforme en hemorrágico, solo sabemos que está muy cercano a las cepas asiáticas aisladas en China en el 2012 y Filipinas en el 2013.

Por lo visto con otros virus emergentes y reemergentes, la llegada del virus CHIKV a Colombia es simple cuestión de tiempo, (actualmente en Venezuela) solo queda prepararnos para un nuevo diagnóstico diferencial y asumir desde ahora la vigilancia eco-epidemiológica y clínica.

\section{REFERENCES}

1. Isabelle Leparc-Goffart, Antoine Nougairede, Sylvie Cassadou, Christine Prat, Xavier de Lamballerie. Chikungunya in the Americas, LANCET 2014; 383:514.

2. Leparc-Goffart I, Nougairede A, Cassadou S, C Prat, Lamballerie X. Chikungunya in the Americas. Lancet 2014; 383: 514.

3. Suchitra Nimmannitya, Scott B. Halstead, Sanford N. Cohen, Mark R. Margiotta. Observations on Hospitalized Patients with Hemorrhagic Fever. Am J Trop Med Hyg November 1969; 18:954-971.
4. Powers A, Logue C. Changing patterns of chikungunya virus: re-emergence of a zoonotic Arboviruses. J Gen Virol 2007; 88, 2363-2377.

5. OPS/CDC. Preparación y respuesta ante la eventual introducción del virus chikungunya en las Américas. Washington, DC; Organización Panamericana de la Salud; Centers for Disease Control and Prevention: 2011.

6. Gomes CA, Marandino R, Pimentel MA, Ribeiro NR.Chikungunya virus infection: report of the first case diagnosed in Rio de Janeiro, Brazil. Revista da Sociedade Brasileira de Medicina Tropical 2012; 45(1):128-129. 